Archived version from NCDOCKS Institutional Repository http://libres.uncg.edu/ir/asu/

\title{
Appalachlyan
}

$\overline{B \text { O O N E, N O R T H C A R O L I N A }}$

\section{Counseling Strategies To Improve Nutrition Care For Rural Appalachian Patients}

\author{
By: Melissa D. Gutschall, Jamie M. Marchetti, and Kyle L. Thompson
}

\begin{abstract}
Culturally sensitive strategies are critical to help the rural Appalachian population prevent and manage chronic diseases. A multiple-methods approach examined specific nutrition strategies used by practitioners and community members $(n=61)$. Strategy subthemes were categorized into 4 themes from the previously established Rural Nutrition Care Model (1) access and resources (budgeting, planning, resources), (2) sociocultural influences (whole-family approach, simple messages, building rapport, avoiding assumptions), (3) traditional foods (gardening, cooking), and (4) health behaviors (small changes, prevention). Practitioners and patients had differing perspectives on the nutrition context of the rural population $(P<.05)$, which provides a further rationale for the importance of culturally sensitive nutrition counseling strategies.
\end{abstract}

Gutschall, Melissa D. PhD, RD; Marchetti, Jamie M. MS, RDN, LD; Thompson, Kyle L. DCN, RDN, LDN, CNSC. (2019). Counseling Strategies to Improve Nutrition Care for Rural Appalachian Patients. Topics in Clinical Nutrition, January/March 2019. Vol. 34, no. 1 - pages 77-87. doi: 10.1097/TIN.0000000000000164. Publisher version of record available at: https://journals.Iww.com/topicsinclinicalnutrition/ Fulltext/2019/01000/Counseling_Strategies_to_Improve_Nutrition_Care.9.aspx 


\title{
Counseling Strategies to Improve Nutrition Care for Rural Appalachian Patients
}

\author{
Melissa D. Gutschall, PhD, RD; \\ Jamie M. Marchetti, MS, RDN, LD; \\ Kyle L. Thompson, DCN, RDN, LDN, CNSC
}

\begin{abstract}
Culturally sensitive strategies are critical to help the rural Appalachian population prevent and manage chronic diseases. A multiple-methods approach examined specific nutrition strategies used by practitioners and community members $(n=61)$. Strategy subthemes were categorized into 4 themes from the previously established Rural Nutrition Care Model (1) access and resources (budgeting, planning, resources), (2) sociocultural influences (whole-family approach, simple messages, building rapport, avoiding assumptions), (3) traditional foods (gardening, cooking), and (4) health behaviors (small changes, prevention). Practitioners and patients had differing perspectives on the nutrition context of the rural population $(P<.05)$, which provides a further rationale for the importance of culturally sensitive nutrition counseling strategies. Key words: Appalachia, interviews, nutrition counseling, rural nutrition, strategies
\end{abstract}

$\mathbf{R}$ URAL CULTURE influences how members of rural populations understand, receive, and access health care, including nutritional care. ${ }^{1}$ Roughly $19.3 \%$ of Americans lived in a rural setting, an area with a population of less than 2500 people per square mile, according to the 2010 US Census. ${ }^{2}$ Aside

\footnotetext{
Autbor Affiliations: Department of Nutrition and Health Care Management, Appalachian State University, Boone, North Carolina (Dr Gutschall and Dr Thompson); and Campbell County Health, Gillette, Wyoming (Ms Marchetti).
}

At the time of study, Ms Marchetti was a graduate student in the Department of Nutrition and Health Care Management at Appalachian State University.

The authors have disclosed that they have no significant relationships with, or financial interest in, any commercial companies pertaining to this article.

Correspondence: Melissa D. Gutschall, PhD, RD, Graduate Program in Nutrition, Department of Nutrition and Health Care Management, Appalachian State University, 1179 State Farm Road, Boone, NC 28607 (gutschallmd@appstate.edu).

DOI: 10.1097/TIN.0000000000000164 from this population-based definition, there is no single definition of rural, but rather a rural-urban continuum based on the degree of exclusion or isolation. ${ }^{3,4}$ The Appalachian region is a rural area defined by its location in the Appalachian mountain range as well as by its shared cultural characteristics among the people of the region. Nearly half ( $42 \%)$ of Appalachian residents live in a rural setting. ${ }^{2}$

The geography of Appalachia and the cultural values of the Appalachian people may discourage individuals from seeking preventative health care. Barriers to seeking health care in rural settings include isolation, low educational levels, low incomes, less accessibility to health care, and fewer health specialists. ${ }^{1}$ The Appalachian community suffers from higher rates of mortality from chronic diseases including obesity, heart disease, cancer, stroke, and diabetes than the overall US population. ${ }^{2,5}$ In fact, health disparities in the region are more severe than in other rural areas nationwide, and life expectancy among Appalachian residents has 
worsened to 2.4 years less than the national average since $1990 .{ }^{6}$ Three of the 5 leading causes of death-heart disease, cancer, and stroke - are directly related to nutritional factors that impact both prevention and treatment. ${ }^{7-9}$

Even with apparent lack of access to sufficient health care for members of this Appalachian population, many are underserved and need access to specialized health services such as nutrition counseling. ${ }^{10,11}$ Inconsistencies in the concentration of access to dietitians among rural areas ${ }^{12,13}$ and recruitment of health professionals to these settings remain challenging. ${ }^{14}$ Appropriate health services remain an issue for rural Appalachian people and warrant investigation of ways to improve preventative health behaviors among this population. Multiple factors have been shown to play a role in the diet-related behaviors of rural Appalachian residents including accessibility and resources for health care, sociocultural characteristics, health beliefs, and traditional dietary patterns. ${ }^{15}$ Residents there identify the need for modified health behaviors and improved quality health care in Appalachia. ${ }^{11,16}$

A complete understanding of the local culture could help serve this population's health care and nutrition needs, ${ }^{17}$ whereas violation of these cultural norms may create additional barriers to meeting those needs. Adequate and accurate nutrition information is critically important in a region plagued by chronic disease. ${ }^{10,18}$ Limited research exists on how dietitians can best address rural Appalachian cultural and behavioral aspects for individuals within the counseling situation. ${ }^{19-22}$ Thus, this study investigated barriers to improve nutritional status and obtain nutrition care as perceived by members of the rural Appalachian community and the dietitians who work with them to discover specific, practical, and culturally sensitive strategies to overcome barriers. The research aims focused on culturally appropriate nutrition education and dietary interventions in rural Appalachia to build an evidence base for quality dietitian services for this population. A secondary purpose of the study used the knowledge gained to improve training of dietetics students for work with rural populations.

\section{METHODS}

\section{Design}

This study used multiple methods to identify potential tactics to help residents in the rural Appalachian community overcome obstacles for improving nutritional status and obtaining nutrition care. This research design applied phenomenology and focus groups as the main qualitative methods. ${ }^{23}$ Individual interviews were used to gain insight into their experiences, perceptions, behaviors, and practices, while focus groups were used to generate group discussion. Quantitative data were collected using numeric responses to questionnaires. These data were used to reinforce findings from qualitative data.

\section{Sample}

Participants were recruited via advertisements at community health facilities as well as through students and preceptors participating in the graduate dietetic internship program associated with the sponsoring institution. Telephone screening was conducted to determine eligibility, explain the interview process, and schedule a meeting for individual interviews.

The participants were composed of registered dietitians (RDs) practicing in rural areas, dietetic interns (DIs) being trained in rural areas, and patients who were adults ( $\geq 18$ years) with a chronic disease who lived in a rural area (territory encompassing less than 2500 people) for a substantial portion of their life (at least one-third). ${ }^{24}$ Phase 1 results were incorporated into the previously published Rural Nutrition Care Model, ${ }^{15}$ and additional data were collected during Phase 2, further probing for strategies to overcome the barriers identified in each component of the model. Data from both phases were combined and analyzed. A total of 20 DIs participated in focus groups. A total of 15 patients and 16 RDs provided interviews. No participant contributed to both phases of the study, and all 
data were triangulated when analyzing for the concept of rural nutrition strategies.

The institutional review board at the sponsoring institution approved this research. All participants provided written informed consent and volunteered to give their time to their respective phase of the study. Participants in both phases received an equal monetary honorarium at the completion of their participation.

\section{Procedures}

The investigator framed the methods for this study with the assistance and guidance of a $\mathrm{PhD}$ nutrition researcher who was skilled in qualitative research methodology. During the first phase of the study, the researchers collaborated to develop a guide for the semistructured interview using pilot data that originated in DI field notes related to practice in rural populations. ${ }^{23,25}$ Four preliminary themes emerged in preliminary data collection and are represented in the Rural Nutrition Care Model. They include access and resources for health care and nutrition information, sociocultural influences on food choices, traditional foods consumed, and health behaviors. ${ }^{15}$

The themes categorized the interview questions related to specific strategies. Openended questions in these categories were incorporated in the interview guide, ${ }^{15}$ with follow-up questions and remarks to prompt clarification and elaboration. During the second phase of research, the interview guide was modified to probe further for rural nutrition strategies with questions such as "What particular strategies do you find most useful when counseling rural patients?". Questions were not pilot tested prior to use; however, questions were based on preliminary data and refined for the second phase of research.

Data collection included two 60-minute focus groups, 1 during each phase with 10 DIs each in a classroom setting. Interviews were 30 to 60 minutes each with patients and practitioners in various settings either in person or via telephone. All of the interviewers conducted the interviews and focus groups with training from an experienced researcher to prevent bias. The focus groups and interviews were recorded and transcribed verbatim for analysis. Transcriptions excluded participant identifiers. Interviews occurred simultaneously with transcription of previous interviews to facilitate constant comparison among the data and ensure data saturation and interrater reliability among investigators. Interviews were completed when data saturation was reached, indicated by a high frequency of repetitive responses and themes from participants.

The participants were also asked to complete a 40-item questionnaire following their interview. Two questionnaires, 1 for patients and 1 for practitioners and interns, were developed to gather data about rural health overall as well as specific demographic information from each participant. Both questionnaires asked the same questions and used Likert scales (1-5) to rate statements in each of the categories of the 4 preliminary themes. Questionnaire responses indicated general impressions of specific aspects of each theme validated in the Rural Nutrition Care Model. ${ }^{15}$ Some sample questionnaire topics can be found in Gutschall et $\mathrm{al}^{16}$ or by writing the lead author of this article.

\section{Data analysis}

Descriptive statistics and independent samples $t$ tests were used to analyze demographic and rural health questionnaires using SPSS (IBM, Version 20, 2012; IBM Corp, Armonk, New York). ${ }^{26}$ Questions were recoded so that patient and practitioner responses aligned to the same ends on the Likert scale (eg, where practitioners responded to the statement, "My rural patients may lack knowledge regarding portion size and menu variety," patients responded to the statement, "I feel I know a lot of information about portion size and menu variety"). When disagreement was indicated by a response of 1 and agreement by a response of 5, patient responses were recoded so that the numeric response was in line with the amount of perceived knowledge for both 
questionnaires and make them comparable for statistical analysis.

Qualitative interviews were transcribed and reviewed to ensure accuracy. The researchers collaborated to find patterns in the interview transcripts, draw conclusions, and reach a consensus on the major indications. Discrepancies on coding and thematic development were resolved via group discussion involving all of the researchers; thus, agreement was reached by investigators on all thematic findings. Data were analyzed to identify themes surrounding nutrition care strategies for rural populations. The transcripts were coded using NVIVO Qualitative Analysis Software (Version 10, 2013; QSR International, Melbourne, Australia).

During the first phase of research, ideas from the data were grouped according to similarity and categorized by theme. Coding, grouping, and thematic analysis of all transcripts were reviewed and agreed upon by 3 researchers. ${ }^{23,25}$ During the second phase, both the previously gathered data and new data were analyzed for information on strategies to help the rural Appalachian population overcome barriers to nutrition care. The findings were triangulated from each of the participant groups to confirm the themes.

\section{Findings}

RDs $(n=17)$ had practice experience ranging from 5 months to 40 years, with a mean of 14.4 years. On average, RDs estimated that $75 \%$ of their patients were rural dwellers. Twenty DIs were completing their second year of a combined graduate program in nutrition and the dietetic internship. Internship rotations were located in rural areas or urban areas with facilities that provided services to nearby rural locations. Patients $(n=24)$ ranged in age from 23 to 80 years (mean of 55 years). All patients had lived in a rural area for at least 22 years. Patients reported chronic diseases, most frequently including heart disease, diabetes, hypertension, and obesity. Six patients reported more than 1 chronic condition.
The results from RDs and DIs were similar and collapsed for analysis, and thus, these data are presented collectively and are referred to as "practitioner" results. The strategic subthemes are presented within each model component in the Table and include the number of mentions by practitioners and patients. Another finding was the difference between practitioners' and patients' responses to questionnaire items, providing some insight to the efficacy of nutrition counseling strategies targeting cultural beliefs and values.

\section{Model component 1: Access and resources}

Three strategic subthemes were identified in component 1 . The largest subtheme was budgeting with 36 mentions. In some instances, budgeting indicated that patients lacked sufficient money or time to buy and prepare nutritious foods. This was illustrated when a 39-year-old male patient described, "Going to the store is time-consuming. It takes a while to get there, depending on how much money you want to spend because the little local grocery store is a little more expensive than one if you were to drive quite a while to get some cheaper rates, yet you're sacrificing time." The remaining 2 themes in this component, planning and resources, had 37 and 27 mentions, respectively. Planning responses suggest that deliberate forethought could help overcome nutrition barriers. During a focus group of 10 DIs completing 9 months of rotations in rural settings, 1 practitioner described a useful planning method, saying, "Teaching them easy ways to cook that food, like crock pot cooking, a lot of them don't use it, but it's a way that they can, they have 10 minutes at night after they do whatever, throw it in and take it and put it in there in the morning, they have a home-cooked meal. A lot of just some of those easy techniques maybe to having those things that aren't labor-intensive at one given time." Availability of information and services as a counterbalance to barriers were categorized as resources. "I think the information that I would definitely provide is, "What do we have available in this area?" stated an RD 
Table. Counseling Strategies Identified by Qualitative Theme and Subtheme

Theme: Access and Resources

\begin{tabular}{ll}
\hline Subtheme & \multicolumn{1}{c}{ Strategy } \\
\hline $\begin{array}{l}\text { Budgeting (36 mentions; } 17 \mathrm{RDs} ; \\
\text { 19 patients) }\end{array}$ & Teach tips and tricks for saving money \\
Planning (37 mentions; $18 \mathrm{RDs} ;$ & Teach how to plan healthy meals and shop ahead \\
19 patients) & Help patients find local organizations or opportunities \\
Resources (27 mentions; $21 \mathrm{RDs} ;$ & for nutrition education or food assistance that will \\
6 patients) & work for them \\
\end{tabular}

Theme: Sociocultural Characteristics

\begin{tabular}{ll}
\hline Subtheme Strategy & Strate \\
\hline
\end{tabular}

Whole-family approach

(36 mentions; 20 RDs;

16 patients)

Simple messages (41 mentions; 31 RDs; 10 patients)

Building rapport and relationships (46 mentions; 40 RDs; 6 patients)

Avoiding assumptions and judgment (27 mentions; 26 RDs; 1 patient)
Counsel multiple family members together, including the food gatekeeper for the family

Use layman's terms and teach to the patient's level of understanding

Listen to patients, express sensitivity to their emotional needs, and perform professionally

Remain open to learning about individuals and their unique situation

Theme: Traditional Foods

Subtheme Strategy

Gardening (38 mentions; 15 RDs;

23 patients)

How to cook (17 mentions; 9 RDs; 8 patients)
Encourage patients to try growing some of their own produce, even a small amount

Explain practical cooking tips that patients can easily implement for healthier cooking

Theme: Health Behaviors

Subtheme

Strategy

Small changes (56 mentions; 37 RDs; 19 patients)

Prevention (16 mentions; 9 RDs; 7 patients)
Guide patients in choosing small, positive steps to take to move them toward healthier behaviors in a way they feel they can manage

Stress the importance of healthy behaviors to prevent health problems later on

Abbreviation: RD, registered dietitian.

who had been practicing in a rural area for 3 years.

Questionnaire items related to this model component provide information about how to go about developing strategic approaches to counseling rural patients. Practitioners and patients disagreed about the availability of full-service grocery stores $(P<.001)$. Practitioners felt that their rural patients often experienced limited access to full-service 
stores, while patients indicated that they usually had a full-service grocery store within 20 miles of their home. In addition, patients and practitioners thought differently about the ease of transportation $(P<.001)$. Patients reported that they did not have trouble arranging transportation, but practitioners indicated that this was often a struggle for their rural patients. Disagreement was also seen in responses to questionnaire items regarding health insurance. Practitioners perceived that their rural patients were less likely to have health insurance, while most patients reported having health insurance $(P<.001)$.

\section{Model component 2: Sociocultural characteristics}

The strategic subthemes identified in this component focused mainly on effective nutrition education methods (see the Table). The theme mentioned most often (36 mentions) was the whole-family approach. This theme encompassed the idea that many rural families have matriarchal gatekeepers who purchase and prepare food, and other members of the family often exert pressure on the matriarchal figure to purchase and prepare favorite, desired, and frequently unhealthy foods. A 30-year-old female patient explained, "We don't have enough money to buy two things like for healthy food and his food. He is not interested in changing his lifestyle at this moment."

The second most-frequent theme in this component was simple messages, with 41 mentions, a theme that emphasized the importance of streamlining nutrition education to avoid confusion and complexity. A practitioner in the DI focus group stressed the significance of "making sure that they know that they don't have to change everything at once. You're focusing on one thing that might be a little bit easier, and if there's more visits, you can work on other things later." Building Rapport and Relationships mentions (46 mentions) indicated that a level of trust and familiarity between patients and practitioners would enable greater nutrition education by increasing the level of comfort of the patient.
"It was kind of embarrassing that I would have a problem that I would have to go to someone to teach me how to eat," explained a 64-yearold female patient. Similarly, although somewhat different, was the theme of avoiding assumptions and judgment with 27 mentions. This theme captured the idea that a practitioner may not understand why patients engage in their nutrition behaviors or habits and thus must allow space to try to understand the individual.

Combining this information with questionnaire findings creates a basis for considering strategy improvement in the sociocultural influences model component. Patients and practitioners agreed that most households have a matriarchal gatekeeper, a woman who oversees the entrance of food into the home by handling the responsibility of doing the grocery shopping $(P=.117)$. However, where practitioners thought women cooked most of the meals, patients indicated this was not always the case $(P=.045)$. With regard to the need for lower literacy education materials, practitioners believed these materials were necessary while patients did not $(P<.001)$. When working to build rapport and relationships, practitioners thought that some rural patients did not trust outsiders. In contrast, patients indicated an overall disagreement to that statement $(P<.001)$.

\section{Model component 3: Traditional foods}

Strategic subthemes in this component were gardening, with 38 mentions, and how to cook, with 17 mentions (Table). These subthemes reflect a return to eating patterns reminiscent of the past that included production and preparation of food in the home. With regard to the subtheme of Gardening, a 62-yearold female patient expounded, "I was raised in these mountains, back up in the mountains, and we always had fresh food. It was something that comes natural, even canning, to this day for me. I think it's a great area to live, a great area to raise fruits and vegetables. We're trying to get an orchard started on the farm we're at now, that type of thing. I want some black raspberries and strawberries, and 
that type of thing. And teach my grandchildren the same thing." The subtheme of How to Cook was reflected in a patient's expression of interest in tips from a dietitian: "Set up some invitations to try some foods and clinics, have a clinic and do some cooking in front of the people and teach them the difference between what you put in the vegetables and what you don't put in, you know, like fat back or something where it's more nutritional. And then indicate why it's better for them."

Questionnaire responses reinforce nutrition counseling strategies in this model component. Practitioners perceived that a number of their rural patients grew their own food, but very few patients reported growing their own food $(P<.001)$. Teaching healthy cooking methods is also supported by the questionnaire results. Patients' questionnaire results showed that they did not cook with high-fat products frequently, while practitioners indicated regularity of high-fat cooking methods used by their patients $(P<.001)$. Practitioners identified a higher level of importance of traditional family foods in the lives of their rural patients than the patients themselves did $(P<.001)$.

\section{Model component 4: Health behaviors}

Two strategic subthemes comprised this component. The first is small changes with 56 mentions. Small changes indicate actions that patients can choose to take to begin from their current practices to a state of improved health and nutrition. A key part of small changes is that the practitioner allows the patient to determine what degree of change seems manageable. A DI explained in the focus group, "Take small steps ... it may take you longer but it'll keep you focused." The second theme in this component (16 times) is prevention, which shows that helping patients improve their nutrition prior to the onset of nutritionrelated diseases may allow patients to avoid the disease and delay remedial treatment or management of the disease in the future. "Oftentimes I hear the quote, 'Well, everybody's going to die from something,' and as a result of that, they continue with their same dietary patterns without really thinking about the consequences," said an RD who had practiced for 19 years in a rural area.

Within this component, questionnaire results lend credence to the qualitative analysis findings. While patients disagreed with practitioners about the rural population's lack of knowledge of a healthy lifestyle $(P<.001)$, both parties reported that overweight and obesity were common among the rural population $(P=.603)$. Patients and practitioners strongly agreed that rural patients tend to wait to seek medical attention until a condition becomes advanced $(P=2.051)$. Patients were more likely than practitioners to indicate adequate access to nutrition education and dietitian services for rural patients $(P=.002)$.

\section{DISCUSSION}

This research project examined barriers to improving nutritional status and obtaining nutrition care as perceived by members of the rural Appalachian community and practitioners who work with them. Data were collected to identify specific, individualized, and culturally sensitive strategies to overcome these perceived barriers. Considering the 4 components of the Rural Nutrition Care Model-access and resources, sociocultural characteristics, traditional foods, and health behaviors-the participants indicated several nutrition counseling strategies to help individuals overcome barriers.

Under access and resources, the project revealed strategies used by practitioners to help patients surmount barriers. Practitioners and patients indicated that strategies focused on budgeting, planning, and resources, and increasing awareness of community resources were the most important in assisting patients to make the most of what they have available. This finding aligns with previous research that has indicated that structure such as grocery stores, available variety, and required travel may inhibit rural residents to improve nutritional status. Prior research also indicated that social networks can help raise awareness of resources such as sales and health fairs 
with free checkups. ${ }^{27}$ Thus, strategies used to increase community-wide knowledge of the means by which to overcome barriers can be improved by reaching individuals who can facilitate the sharing of this information. Additional research has shown that fast foods are increasingly found in nontraditional fast-food outlets such as convenience stores and grocery stores in rural areas. Thus, the need to teach patients how to make better nutrition choices with the increased availability of fast foods could benefit rural residents. ${ }^{28}$

This discrepancy indicates a need for strategies that focus not only on making stores available within easy travelling distance, but on ways to take advantage of access to full-service grocery stores when patients are able to visit them. Different responses on insurance topics illustrated that while many rural patients are insured, their needs may not all be covered, and many may lack coverage altogether. Thus, continuing to make rural patients aware of community resources where they can find health assistance remains an important strategy.

For sociocultural characteristics, the respondents gave strategies used by practitioners to connect with their patients and share messages. Strategies to reach patients included using a whole-family approach, simple messages, building rapport and relationships, avoiding assumptions and judgment, and interactive learning. The goal of these strategies is to work within rural social contexts including matriarchal gatekeepers, distrust of outsiders, and necessity of low-literacy education materials. These strategies are supported by earlier research indicating greater effectiveness of messages from health care professionals when those messages are presented with adherence to: privacy concerns; values, beliefs, and customs; literacy needs; and family characteristics of patients. ${ }^{29}$

Questionnaire results on the topics of matriarchal gatekeeper and women cooking lend credence to the whole-family approach of nutrition counseling. If the matriarchal gatekeepers are aware of the nutritional needs of all family members, they will be more likely to purchase appropriate foods. Subsequently, once in the home, any family member can prepare healthy food. The questionnaires also highlighted an inconsistency in the perception of need for lower literacy education materials. This perception could be indicative of the existing use of low-literacy education materials that has, from the perspective of the patient, masked the necessity of using the lowliteracy education materials because patients are not aware that the materials are simplified or how to use them.

Although educational attainment for rural populations has improved, approximately $17 \%$ of the rural population older than 25 years have not earned a high school diploma, reinforcing the need for low-literacy nutrition education materials. ${ }^{30}$ Another contradiction found from the questionnaire data was on the level of trust of outsiders. Members of the rural population may trust outsiders if they are met in the community yet might have a different attitude toward health care practitioners. ${ }^{17}$ If this is the case, persistence on the part of practitioners to build strong rapport with their patients is important as a nutrition counseling strategy.

This project brought some strategies to light within the component of traditional foods that may be effective for accommodating the food preferences of rural patients while improving the nutritional value of those foods. Gardening and teaching healthful cooking methods may help rural patients make better food choices while continuing to incorporate traditional foods that are culturally meaningful. Reviving the cultural heritage of foods (ie, traditional vegetable dishes) may be a potentially strong motivator for dietary behavioral change. Past research indicates that community-based intervention where the target population participates in developing the intervention is likely an effective way to facilitate the implementation of nutrition changes. ${ }^{31}$ In addition, the messages for these changes are more effective if shared and tailored to the population rather than presented as threatening messages based on to the consequences of poor health behaviors. ${ }^{32}$ These 
factors should be kept in mind when making recommendations that are culturally sensitive to food traditions and worthwhile for patients to incorporate into their lifestyles.

Conflicting questionnaire results on the topic of self-provision of food showed that encouragement of growing even a little of one's own food is a useful nutrition counseling strategy. Also, data from questionnaires regarding high-fat cooking methods and importance of traditional family foods indicated that teaching healthier cooking methods through minimizing high-fat products and improving the nutritional value of family favorite recipes supported how to cook as a nutrition counseling strategy. Although patients reported less importance of traditional recipes than practitioners perceived, the ability to prepare these foods using healthful techniques would still benefit patients.

In the Health Behaviors section of the Rural Nutrition Care Model, key strategies emerged. Small changes and prevention were the most noticeable strategies. Both patients and practitioners recognized that overweight and obesity are widespread throughout the rural population and that patients in these areas are likely to wait to seek medical attention. There was a disconnect between the perspectives of patients and practitioners regarding community knowledge of healthy lifestyles and access to nutrition education resources. Making small changes, enabled through mentoring by peers, can improve preventative health behaviors. ${ }^{33}$ In addition, working with the existing paradigm held by the population regarding what constitutes health and working to align that concept of health with a biomedical model could open the door to altering health behaviors of rural patients.

Rural Appalachian patients view health as the ability to function in the community rather than just avoiding illness. ${ }^{22}$ Thus, relating particular diet changes to specific health outcomes may help this population recognize the role of nutrition in maintaining functionality in a community.

Inconsistencies were demonstrated by patients from the qualitative analysis regarding knowledge of healthy lifestyles and the rates of overweight and obesity in the rural population. Perhaps this indicates that patients in the rural area are aware of what a healthy lifestyle should be but are uncertain on how to incorporate healthy behaviors into their own lives. Using the small changes subtheme to inform a nutrition counseling strategy can help patients move toward a healthier lifestyle in ways that are tailored to the individual. This focus reinforces prevention as a nutrition counseling strategy also revealed in the questionnaire results. Questionnaire data on 2 items, delay in seeking medical attention and adequacy of access to nutrition education and dietitian services for rural patients, can be considered together. It is possible that, even though patients think they have access to nutrition education and dietitian services, they are not necessarily aware of how the services relate to their health and the prevention of medical conditions.

While this qualitative study included a small number of participants, the participants were carefully selected as key informants to provide an accurate, insightful depiction of the rural Appalachian culture, as it pertains to nutrition and nutrition care. Although the interviews varied, researchers used techniques that were necessary and appropriate to obtain enough data for saturation and adequate representation of rural areas. The triangulation of the results from RDs, DIs, and patients also provided some strength to the design. Since this research was focused on the Appalachian area, the findings may not be generalizable to all rural populations.

\section{CONCLUSIONS}

This research provides support for culturally sensitive nutrition counseling strategies for rural Appalachian patients. The differing perspectives from which patients and practitioners approach nutrition counseling can be the basis for further work. The strategies that were predominantly RD driven related to their work and relationships with patients. The strategies that were predominantly 
patient driven included hands-on practical skills related to daily food. Further research is needed to demonstrate the effectiveness of these strategies, particularly among subsets of the population grouped by disease state or degree of rurality. The project could be applied to other rural populations and tested to adapt these strategies. To take hold, increased access to and awareness of RDs may need to take place first. There is a need for RDs who are trained to work in rural areas. Improved community outreach and accessibility may help bridge the gap between patients and nutrition care. The scope of this study was not far enough to capture the extremely rural population that is isolated due to lack of transportation, severe distrust, without adequate access to resources, or other reasons. Differences in the degree of remoteness and the severity of these outcomes were evident throughout the research. Improved access to nutrition care for those who are isolated could have a ripple effect within the rural population as a whole.

\section{REFERENCES}

1. Farmer J, Bourke L, Taylor J, et al. Culture and rural health. Aust J Rural Health. 2012;20(5):243-247.

2. United States Census Bureau. 2010 Census Urban and Rural Classification and Urban Area Criteria. Washington, DC: United States Census Bureau; 2014. http://www.census.gov/geo/reference/urban-rural2010.html. Accessed May, 192016.

3. Miller K. Rural definitions. http://www.rupri.org/ Forms/RuralDefinitions.pdf. Accessed 2017, July 21.

4. United States Department of Agriculture Economic Research Service. Rural-Urban Continuum Codes. https://www.ers.usda.gov/data-products/rural-urbancontinuum-codes/. Published 2013. Accessed July $12,2017$.

5. Phillips CD, McLeroy KR. Health in rural America: remembering the importance of place. Am J Public Health 2004;94(10):1661-1663.

6. Singh GK, Kogan MD, Slifkin RT. Widening disparities in infant mortality and life expectancy between Appalachia and the rest of the United States, 19902013. Health Aff. 2017;36(8):1423-1432.

7. Academy of Nutrition and Dietetics Evidence Analysis Library. Heart Failure Guideline. https://www. andeal.org/topic.cfm?menu=5289. Published 2017. Accessed August 28, 2017.

8. Academy of Nutrition and Dietetics Evidence Analysis Library. Oncology Guideline. https://www.andeal .org/topic.cfm?menu=5291. Published 2013. Accessed August 28, 2017.

9. Academy of Nutrition and Dietetics Evidence Analysis Library. Hypertension Guideline. https://www. andeal.org/topic.cfm?menu $=5285 \&$ cat $=5583$. Published 2015. Accessed August 28, 2017.

10. Behringer B, Friedell GH. Appalachia: where place matters in health. Prev Chron Dis. 2006;3(4):113.

11. Denham SA, Meyer MG, Toborg MA, Mande MJ. Providing health education to Appalachia populations. Holist Nurs Pract. 2004;18(6):293-301.

12. Occupational Employment and Wages, May 2017; 29-1031 Dietitians and Nutritionists. U.S. Bureau of Labor Statistics, Division of Occupational Employ- ment Statistics. https://www.bls.gov/oes/current/ oes291031.htm. Updated March 30, 2018. Accessed May 24, 2018.

13. Rural Health Information Hub. Why Diabetes is a Concern in Rural Communities. https://www.rural healthinfo.org/toolkits/diabetes/1/rural-concerns. Published 2018. Accessed May 24, 2018.

14. McGrail MR, Wingrove PM, Petterson SM, Bazemore AW. Mobility of US rural primary care physicians during 2000-2014. Ann Fam Med. 2017;15(4):322328.

15. Gutschall MD, Thompson KL, Lawrence E. Addressing Health disparities in rural nutrition practice: a qualitative model from rural Appalachia. J Hunger Environ Nutr. 2017;13(1):84-89.

16. Schoenberg NE, Howell BM, Swanson M, Grosh C, Bardach S. Perspectives on healthy eating among Appalachian residents. J Rural Health. 2013;29 (suppl 1):s25-34.

17. Keefe SE. Appalachian Cultural Competency: A Guide for Medical, Mental Health, and Social Service Professionals. 1st ed. Knoxville, TN: University of Tennessee Press; 2005.

18. Borak J, Salipante-Zaidel C, Slade MD, Fields CA. Mortality disparities in Appalachia.J Occup Environ Med. 2012;54(2):146-156.

19. Gustafson AA, Lewis S, Wilson C, Jilcott-Pitts S. Validation of food store environment secondary data source and the role of neighborhood deprivation in Appalachia, Kentucky. BioMed Central Public Health. 2012;12:12.

20. Wilson SL, Kratzke C, Hoxmeier J. Predictors of access to healthcare: what matters to rural Appalachians? Global J Health Sci. 2012;4:23-35.

21. McGarvey EL, Leon-Verdin M, Killos LF, Guterbock T, Cohn WF. Health disparities between Appalachian and non-Appalachian counties in Virginia USA. $J$ Community Health. 2011;36(3):348-356.

22. Goins RT, Spencer SM, Williams K. Lay meanings of health among rural older adults in Appalachia.J Rural Health. 2011;27:13-20. 
23. Green J, Thorogood N. Qualitative Methods for Health Research. 3rd ed. London, UK: Sage Publications; 2013.

24. United States Census Bureau. 2010 Census Urban and Rural Classification and Urban Area Criteria. https:// www.census.gov/geo/reference/ua/urban-rural-2010 .html. Published 2010. Accessed April 16, 2015.

25. Noble $\mathrm{H}$, Smith $\mathrm{J}$. Issues of validity and reliability in qualitative research. Br Med J Evid Based Nurs. 2015;18:34-35

26. de Winter JCF, Dodou D. Five-point Likert items: t test versus Mann-Whitney-Wilcoxon, Pract Assessment Res Eval. 2010;15(11).

27. Ramadurai V, Sharf BF, Sharkey JR. Rural food insecurity in the United States as an overlooked site of struggle in health communication. Health Commun. 2012;27(7):794-805.

28. Creel JS, Sharkey JR, McIntosh A, Anding J, Huber JJC. Availability of healthier options in traditional and nontraditional rural fast-food outlets. BMC Public Health. 2008;8:395.
29. Bushy A. Conducting culturally competent rural nursing research. Annu Rev Nurs Res. 2008;26:221236.

30. USDA Economic Research Service. Educational attainment for people age 25 and older, 2000 and 2007 11. July 26, 2013. http://www.ers.usda.gov/dataproducts/chart-gallery/detail.aspx? chartId=39268. Accessed April 16, 2013.

31. Sloane DC, Diamant AL, Lewis LB, et al. Improving the Nutritional resource environment for healthy living through community-based participatory research. J Gen Intern Med. 2003;18(7):568-575.

32. Kessels LTE, Ruiter RAC, Brug J, Jansma BM. The effects of tailored and threatening nutrition information on message attention. Evidence from an event-related potential study. Appetite. 2011;56(1): 32-38.

33. Smith LH, Holloman C. Comparing the effects of teen mentors to adult teachers on child lifestyle behaviors and health outcomes in Appalachia. J Sch Nurs. 2013;29(5):386-396. 